\title{
RING NEBULAE AROUND MASSIVE STARS
}

\author{
YOU-HUA CHU \\ Astronomy Department, University of Illinois \\ 1002 W. Green Street, Urbana, Illinois 61801 \\ U. S. A.
}

\begin{abstract}
Ring nebulae have been found around WR stars, OB and of stars, and luminous blue variables. Ring nebulae are formed by the interaction between the central stars and their ambient medium via different combinations of stellar winds, ejecta, and radiation. The spectral properties of the nebulae can be used to diagnose the stellar properties, such as luminosity and effective temperature. Correlations between ring nebulae and their central stars may be used to check scenarios of stellar evolution.
\end{abstract}

\section{INTRODUCTION}

"Ring nebulae around massive stars" could be the plural form of "ring nebula around massive stars" or "ring nebula around a massive star".

In the former case, the central stars could be one or multiple OB associations or clusters, and the ring nebula would be a superbubble blown by the stellar winds and supernova remnants jointly. Examples are Loop I in our Galaxy (Weaver 1977), N51D and N70 in the Large Magellanic Cloud (LMC). The size of such ring nebulae ranges from a few tens pc to hundreds or even thousands of pc, and their expansion velocities are usually below $50 \mathrm{~km} / \mathrm{s}$, with the majority being 10-25 km/s (Georgelin et al. 1983; Rosado 1986). Ring nebulae seen in external galaxies are mostly of this type. These nebulae provide excellent labs for studying the deposition of stellar energies into the interstellar medium and they provide diagnostic information for the integrated stellar properties. However, these applications are not immediately relevant to the theme of this symposium; therefore, I will not cover ring nebulae around OB associations any further, and from now on my "ring nebulae around massive stars" would mean strictly one dominant central massive star in each nebula.

About 25 years ago, a ring-shaped nebula would have to be either a planetary nebula or a supernova remnant. When Johnson and Hogg (1965) found S308, as well as NGC 2359 and NGC 6888, was a ring-shaped nebula centered on a WR star, they suggested that WR stars could interact with their ambient medium to form ring nebulae. Subsequent searches around

K. A. van der Hucht and B. Hidayat (eds.),

Wolf-Rayet Stars and Interrelations with Other Massive Stars in Galaxies, 349-363.

() 1991 IAU. Printed in the Netherlands. 
known WR stars in our Galaxy and the Magellanic Clouds indeed turned up many more ring nebulae, thus establishing "ring nebulae around WR stars" a new class of objects. Ring-shaped nebulae are also found around other types of massive stars, such as of stars and luminous blue variables. Originally ring nebulae were recognized morphologically; however, some nebulae that are known to be shaped by winds or consist of stellar ejecta have been referred to as "ring nebulae," although their morphologies are quite irregular.

In this review, I will first describe the different types of ring nebulae, discuss their formation mechanisms, and use them to diagnose stellar properties.

\section{IDENTIFICATION OF RING NEBULAE}

Ring nebulae have been identified in a variety of ways. The classical method applies the criterion of ring- or arc-shaped morphology to the optical images. When the IRAS all-sky survey became available, the infrared images were used efficiently to search for bow shocks around $O B$ stars and ring nebulae around WR stars. A few HI shells or cavities were found around WR stars from the HI $21 \mathrm{~cm}$ line observations. Some ring nebulae are well-known stellar ejecta.

\subsection{Ring Nebulae around WR Stars}

The first three known WR ring nebulae, NGC 2359, NGC 6888, and S308, are all in the north (Johnson and Hogg 1965). Smith (1967) surveyed the galactic WR stars and found four ring nebulae in the south - NGC 3199, RCW58, RCW78, and RCW104. Several incidental discoveries were reported by, for example, Crampton (1971), Johnson (1975), Lortet, Niemela, and Tarsia (1980). Two systematic surveys for the 159 stars in the Sixth Catalog of Galactic WR Stars (van der Hucht et al. 1981) were carried out independently by Chu (1981) and Heckathorn, Bruhweiler, and Gull (1982). Since different investigators had different selection criteria, some reported ring nebulae actually contain several early type stars and some other ring nebulae are not convincingly associated with their WR stars. Chu, Treffers, and Kwitter (1983) re-examined all reported WR rings and concluded, in their Table 2, 15 probable cases and 3 possible cases. An additional double shell structure was later found around the binary system CV Ser (WC8 + 08-9V-III) (González and Rosado 1984). The sizes of these WR rings are mostly in the range of a few pc to $30 \mathrm{pc}$.

Neutral hydrogen holes or shells have been reported around four WR stars: HD 113904 ( $\theta$ Mus), HD 88500, HD 156385, and HD 197406 (Cappa de Nicolau and Niemela 1984; Cappa de Nicolau, Niemela, and Arnal 1986; Cappa de Nicolau et a1. 1988; Dubner et al. 1990). The three WC stars have large HI shells with diameters $-100 \mathrm{pc}$; while the WN7 star, HD 197406, has a much smaller ( $7.6 \mathrm{pc})$ HI hole centered on the star. The expansion velocities are all $\leq 10 \mathrm{~km} / \mathrm{s}$. More HI shells around WR stars are reported by Niemela and Cappa de Nicolau in this volume.

WR rings often stand out against the background better in the IRAS $60 \mu \mathrm{m}$ images, because the IR emission from the dust in the nebula is 
characterized by a higher color temperature than those in the background due to the heating from the central WR star. This effect is best demonstrated by comparing the appearance of S308 on the Palomar Sky Survey Prints, in the [O III] line (Chu et al. 1982), and in the IRAS 60 $\mu \mathrm{m}$ band (Van Buren and McCray 1988). The IRAS survey is being used to search for IR rings around WR stars by Nichols-Bohlin and Fesen (1990). WR ring nebulae have also been searched for in the nearby galaxies. Ten WR rings have been identified in the LMC: DEM 39, 45, 137, 165, 174, 208, 231, 240, and 315, and N79W (Chu and Lasker 1980; Chu 1983a; Rosado 1986). A probable new ring nebula in the LMC, N82, that may contain ejecta from a WC9 star is recently reported by Heydari-Malayeri, Melnick, and Van Drom (1990). No WR stars in the Small Magellanic Cloud have ring nebulae. The search for WR rings has been extended to M33 recently; Drissen, Shara, and Moffat (1990) find 11 probable cases and 8 less likely cases in M33. The WR rings in the LMC and M33 are larger than their galactic counterparts. Part of this discrepancy in size is due to an observational effect: the largest extragalactic WR rings $(>100$ pc) may contain uncataloged $O B$ associations and the smallest WR rings $(<5 \mathrm{pc})$ are yet to be discovered. The median of the smaller WR rings in the LMC or M33 is still larger than the galactic median; this may be due to differences in the interstellar condition among these galaxies.

The smallest ring nebulae are probably the circumstellar shells around the three ofpe/WN9 stars in the LMC: Sk-67 266 , HDE 269858, and HDE 269227 (Walborn 1982). Only the first two nebulae have been resolved spatially (Stahl 1987). The central stars of these nebulae may be LBV's, as described later.

\subsection{Ring Nebulae around of Stars}

Lozinskaya (1982) and Lozinskaya, Lar'kina, and Putilina (1984) surveyed 109 of stars, and found 13 ring nebulae; however, 7 of these rings have more than one 0 star within. The remaining six are the Bubble nebula (NGC 7635), NGC 6164-5, S22, S119, and two dust shells around $\lambda$ Cep and HD 153915, respectively. Miranda and Rosado (1987) suggested two additional possible ring nebulae around HD 313864 and HD 172175.

\subsection{Bow Shocks around OB Stars}

A mass-losing star moving supersonically through the interstellar medium may form a bow shock. Very few bow shocks have been discovered in the optical wavelengths. Two examples are the $09.5 \mathrm{~V}$ star $50 \mathrm{ph}$ (Gull and Sofia 1979) and the $05 \mathrm{~V}$ star $\mathrm{BD}+50^{\circ} 886$ (Deharveng, Israel, and Maucherat 1976; Dyson 1977). Using the IRAS SkyFlux maps, Van Buren and McCray (1988) find 10 additional bow shocks around nearby OB stars.

\subsection{Ring Nebulae around Luminous Blue Variables}

AG Car was once classified a planetary nebula, but the central star is now known to be a luminous blue variable (LBV). $\eta$ Car is surrounded by rapidly expanding ejecta, and the star may be an extreme LBV. P Cygni is surrounded by extended ( $30^{\prime \prime}$ radius) radio emission (Baars and 
Wendker 1987), but no optical counterpart has been found (Stahl 1989). Two of Walborn's (1982) Ofpe/WN9 stars in the LMC are related to LBVs: R127 ( $\equiv$ HDE 269858) is a confirmed LBV (Stahl et al. 1983), and Sk- $67^{\circ} 266$ is a spectroscopic twin to R127 in minimum (Walborn 1982). The circumstellar shells of these two stars are resolved (Stahl 1987); their [N II] line profiles are split by 31 and $38 \mathrm{~km} / \mathrm{s}$, respectively (Walborn 1982).

\subsection{Ring Nebulae around B stars}

Six B stars are found to be surrounded by bow shocks or bow waves (Van Buren and McCray 1988). $\alpha$ Sco B (B2.5V) is found to be in a nebula that is rich in [Fe II] line but weak in $\mathrm{H} \alpha$ and absent in [ $\left[\begin{array}{ll}0 & \mathrm{I}\end{array}\right],\left[\begin{array}{ll}0 & \mathrm{II}\end{array}\right]$, [N II], and [S II] lines (Swings and Preston 1978)! LSS 3027 (B2V) is surrounded by a parabolic-shaped reflection nebula, which is best seen in the blue continuum and absent in the $\mathrm{H} \alpha$ line (Chu 1983b). The arcshaped nebula "Herbig 8" around the B star HDE 250550 (Johnson 1982) is brighter in the blue than in the red, hence may be another reflection nebula (Herbig 1960).

\section{FORMATION MECHANISMS OF RING NEBULAE}

Massive stars can interact with their ambient medium via stellar winds, ejecta, and UV radiation. A ring nebula is presumably formed by a combination of these three modes of interaction.

The first attempt to determine the formation mechanism for a large number of ring nebulae was carried out by Chu and her collaborators, who studied the morphology and internal motion of all known WR rings in the Galaxy and the LMC (Chu 1981, 1982a, b, 1983a; Chu et al. 1982; Chu and Treffers 1981a, b; Chu, Treffers, and Kwitter 1983; Treffers and Chu $1982 \mathrm{a}, \mathrm{b})$. The dynamic timescale of a WR ring nebula, approximated as 0.5 (radius/expansion velocity), is compared to the lifetime of a WR phase. If the nebular dynamic timescale is larger than the WR lifetime, the nebula is classified R-type, for which the central WR star is only responsible for ionizing but not shaping the nebula. For the nebulae with dynamic timescales smaller than the WR lifetime, their nebular properties are compared to those predicted by wind-blown bubble models (Weaver et al. 1977). A nebula is classified E-type if it consists of mostly stellar ejecta, and $W$-type if it is shaped by stellar winds.

One major limitation of this kinematics-based classification is its incapability of determining whether a W-type nebula consists of stellar or interstellar material. Recent analysis of elemental abundances and nebular excitation shows that some of the $W$-type nebulae actually contain shells of stellar ejecta. The classification into E- and Wtypes is apparently overly simplistic and may be somewhat misleading. I will therefore emphasize below the three modes of interaction, instead of the three types of nebulae, and the discussion is generalized to nebulae around other kind of massive stars. Table 1 gives

representative examples for different formation mechanisms; the star names, spectral types, and nebula names are listed. 
TABLE 1. Ring Nebulae around Massive Stars

\begin{tabular}{|c|c|c|c|}
\hline STELLAR EJECTA: & $\begin{array}{l}\text { BAC } 209 \\
\text { AG Car } \\
\eta \text { Car } \\
\alpha \text { Sco B }\end{array}$ & $\begin{array}{l}\text { WN8 } \\
\text { LBV } \\
\text { LBV } \\
\text { B2.5V }\end{array}$ & $\begin{array}{l}\text { M1-67 } \\
\text { AG Car } \\
\eta \text { Car } \\
\text { anon }\end{array}$ \\
\hline BUBBLE/EJECTA : & $\begin{array}{ll}\text { HD } & 147419 \\
\text { HD } & 192163 \\
\text { HD } & 96548 \\
\text { HD } & 148937\end{array}$ & $\begin{array}{l}\text { WN4 } \\
\text { WN6 } \\
\text { WN8 } \\
06.5 \mathrm{fp}\end{array}$ & $\begin{array}{l}\text { RCW104 } \\
\text { NGC } 6888 \\
\text { RCW58 } \\
\text { NGC } 6164-5\end{array}$ \\
\hline WIND-BLOWN BUBBLE: & $\begin{array}{l}\text { HD } 56925 \\
\text { HD } 50896 \\
\text { HD } 89358 \\
\text { HD } 92809 \\
\text { LSS } 4368 \\
\text { BD+60 } 2522\end{array}$ & $\begin{array}{l}\text { WN4 } \\
\text { WN5 } \\
\text { WN5 } \\
\text { WC6 } \\
\text { W0 } \\
\text { 06.5III }\end{array}$ & $\begin{array}{l}\text { NGC } 2359 \\
\text { S308 } \\
\text { NGC } 3199 \\
\text { anon(MR26) } \\
\text { G2.4+1.4 } \\
\text { NGC } 7635 \text { (Bubble Nebula }\end{array}$ \\
\hline BOW SHOCKS: & $\begin{array}{ll}\text { BD }+50^{\circ} 886 \\
\lambda & \text { Cep } \\
\lambda & \text { Ori } \\
\tau & \text { CMa } \\
\alpha & \text { Cam } \\
\zeta & \text { Oph } \\
\kappa & \text { Cas } \\
\delta & \text { Sco } \\
\delta & \text { Pic }\end{array}$ & $\begin{array}{l}05 \\
\text { 06If } \\
\text { OIIIf } \\
09 \text { Ib } \\
09.5 \text { Iae } \\
09.5 \mathrm{~V} \\
\text { BIae } \\
\text { B0.3IV } \\
\text { B3III+09V }\end{array}$ & S206 \\
\hline BOW WAVES: & $\begin{array}{l}\text { HD } 171491 \\
\delta \text { Per } \\
6 \text { Cep }\end{array}$ & $\begin{array}{l}\text { B5 } \\
\text { B5III } \\
\text { B3IV }\end{array}$ & \\
\hline HII REGIONS: & $\begin{array}{ll}\text { HD } & 187282 \\
\text { MR } 97 \\
\text { HD } & 117688 \\
\text { HD } & 115473 \\
\text { HD } & 113904 \\
68 & \text { Cyg }\end{array}$ & $\begin{array}{l}\text { WN4 } \\
\text { WN8 } \\
\text { WN8 } \\
\text { WC5 } \\
\text { WC6+09.5I } \\
07.5\end{array}$ & $\begin{array}{l}\text { anon(MR95) } \\
\text { L69. } 8+1.74 \\
\text { RCW78 } \\
\text { anon(MR46) } \\
\text { anon( } \theta \text { Mus) } \\
\text { S119 }\end{array}$ \\
\hline
\end{tabular}

\subsection{UV Radiation}

A ring nebula that is ionized but not shaped by the central WR star can be identified by its large dynamic timescale. The classification of type $R$ is still meaningful. The R-type nebulae, consisting mostly of interstellar material, are not expected to show any abundance anomaly. The abundances in the R-type nebula RCW78 are indeed similar to those of 
its local interstellar environment (Esteban et al. 1990a).

In retrospect, most of the R-type WR ring nebulae would not have been identified, if the selection criteria had been stricter with respect to filamentary morphology, since a large dynamic age is usually a result of slow expansion, which is implicitly guaranteed by the lack of narrow filaments and sharp limb-brightening on a sub-parsec scale.

For the non-WR central stars, an R-type ring nebula can also be diagnosed by the amorphous nebular morphology, but its confirmation needs more than a comparison of timescales as for the WR rings. For example, the ring nebula 5119 around the of star 68 Cyg, appearing quite amorphous on the Palomar Sky Survey Prints, is recently demonstrated to be an R-type nebula instead of a wind-blown bubble with a central cavity around the star, because the IR emission detected in the vicinity of the star implies the existence of dust, and a bow shock around the star is seen in the IR (Wisotzki and Wendker 1989).

The arc-shaped reflection nebulae around $B$ stars may be related to the UV radiation: the radiation pressure pushes the dust out while the ionized gas is relatively unaffected. The B2V star LSS 3027 gives a good example: its reflection nebula is exterior to the round HII region centered on the star (Chu 1983b).

\subsection{Stellar Ejecta}

"Stellar ejecta" refers to the bulk ejection of stellar surface material, as opposed to the tenuous, continuous, high-velocity stellar winds. Some stellar ejecta are present in the form of a shell, some appear to be bipolar, and some seem to be ballistic clumps. The ejecta nature was originally identified by the kinematic and morphological properties; however, it seems that anomalous abundances may be another good indicator for the ejecta.

Four WR ring nebulae are known to contain stellar ejecta: M1-67, NGC 6888, RCW58, and RCW104. The ejecta nature of M1-67 is concluded from the similarity between the stellar and nebular velocities, which are more than $150 \mathrm{~km} / \mathrm{s}$ higher than the interstellar velocity expected in the galactic rotation (Treffers and Chu 1982a). The shells of ejecta in RCW58 and NGC 6888 are diagnosed from the nebular morphology and excitation; a [N II]-bright ring enveloped in a [0 III]-bright ring can only be explained by a shell of ejecta inside a wind-blown bubble (Chu 1982a; Dufour 1989a; Mitra 1990). The ejecta in RCW104 appear as highvelocity ( $>100 \mathrm{~km} / \mathrm{s})$, [N II] -bright knots near the western rim (Goudis, Meaburn, and Whitehead 1988).

It was once claimed that stellar ejecta were characterized by chaotic internal motion (Chu 1981, 1982a; Treffers and Chu 1982a); however, that claim is apparently an incorrect interpretation of the large aperture Fabry-Perot data of M1-67 and RCW58. Recent long-slit spectra of M1-67, RCW58 and NGC 6888 show smooth bow-shaped line images, indicating regular expansion patterns (Solf and Carsenty 1982; Chu 1988; Marston and Meaburn 1988). The expansion velocities of RCW58 and NGC $6888,110 \mathrm{~km} / \mathrm{s}$ and $80 \mathrm{~km} / \mathrm{s}$, are the highest two among the WR rings.

All of the above four WR nebulae show $\mathrm{He} / \mathrm{H}$ and $\mathrm{N} / \mathrm{O}$ ratios significantly higher than those in the sun and the galactic HII regions 
(see Table 2). Are enhanced $\mathrm{He} / \mathrm{H}$ and $\mathrm{N} / \mathrm{O}$ ratios a sufficient condition for the presence of stellar ejecta? Among the WR ring nebulae in Table 2 , S308 is the only other nebula that shows similar abundance anomalies. It has been argued that S308 must consist of stellar material because of its large distance to the galactic plane (Lozinskaya 1983; McCray 1983). Furthermore, even if $\mathrm{S} 308$ is a bubble blown out of the interstellar material, the stellar wind is not likely to mix with the interstellar material to cause the observed abundance anomaly (Weaver et al. 1977; Dyson and Smith 1985). Therefore, it is very likely that S308 contains mostly stellar ejecta and the $\mathrm{He} / \mathrm{H}$ and $\mathrm{N} / \mathrm{O}$ abundance ratios are indeed an indicator of stellar ejecta.

Among all the ring nebulae around of stars, NGC 6164-5 is the only one that shows definite stellar ejecta, as the $\mathrm{S}$-shaped nebula has both anomalous abundances (Leitherer and Chavarria-K. 1987; Dufour, Parker, and Henize 1988) and kinematics that cannot be explained by wind-blown bubbles (Pismis 1974).

The ring nebulae around the LBV's all consist of ejected stellar material. The nebula around $\eta$ Car has many unique properties; its expansion velocity reaches over $2000 \mathrm{~km} / \mathrm{s}$ (Walborn, Blanco, and Thackeray 1978; Walborn and Blanco 1988; and references therein), and its oxygen abundance is severely depleted while nitrogen abundance is enhanced (Davidson et al. 1986). The nebula around AG Car is relatively moderate with an expansion velocity of $\sim 70 \mathrm{~km} / \mathrm{s}$ (Smith 1990); both oxygen and sulphur abundances are depleted (Mitra and Dufour 1990).

\subsection{Stellar Winds}

The stellar wind from a massive star can sweep up the ambient medium and form a bubble; the motion of the star relative to the medium can cause the star to be decentered (Weaver et al. 1977), and in the extreme case a bow shock, instead of a closed bubble, will be formed (Gull and Sofia 1979). NGC 3199 is an example that the central WR star is probably moving at about $60 \mathrm{~km} / \mathrm{s}$ toward the bright shell ridge at the southwest (Dyson and Ghanbari 1989). The bow shocks around OB stars (Van Buren and McCray 1988) are certainly the results of large proper motions and stellar wind interactions.

Ring nebulae that are formed by stellar winds should show shocks. The [0 III]-bright she11 in RCW58 has been suggested to represent the shock fronts (Chu 1982a). The recent imaging spectral analysis and spectroscopic observations with high spatial resolution show the shocks convincingly for the first time in the Bubble nebula, NGC 2359, and NGC 6888 (Jernigan 1988; Mitra 1990; Dufour 1989a): the [O III] 5007/H $\beta$ intensity ratio reaches 15 and 20 , and the electron temperature may be as high as 25,000 and $40,000 \mathrm{~K}$ for NGC 2359 and NGC 6888, respectively!

Almost all wind-blown bubbles that have been spectroscopically studied have normal abundances relative to their ambient interstellar environments. Examples of the WR rings are NGC 2359, NGC 3199, and the nebula around MR26 (Esteban et al. 1990a; Rosa and Mathis 1990); examples of rings around of stars are the Bubble nebula and the filamentary bubble in NGC 6164-5, located between the S-shaped ejecta and the outermost dust halo (Jernigan 1988; Leitherer and Chavarría-K. 
1987; Dufour, Parker, and Henize 1988). The only exception is S308, which may consist of stellar ejecta, as described in section 3.2.

\section{APPLICATIONS OF THE RING NEBULAE}

The value of ring nebulae around massive stars goes far beyond their apparent beauty. They provide great opportunities to study the central stars as well as the interstellar environment.

\subsection{Nebular Abundances and Stellar Nucleosynthesis}

Ring nebulae may carry processed stellar material, and their abundance anomalies will give information about stellar nucleosynthesis. It is long known that some WR rings are enriched in He and N (Parker 1978; Kwitter 1981, 1984). Recent observations have extended to many fainter nebulae; more importantly, great observational efforts have gone into differentiating the ring nebulae and their interstellar environments so that the abundances in the rings can be compared to the true local interstellar abundances (Esteban and Vilchez 1990; Esteban et al. 1990a; Dufour, Parker, and Henize 1988; Mitra 1990; Jernigan 1988). Table 2 summarizes the recent results for a large number of ring nebulae.

It is clear from Table 2 that the rings containing stellar ejecta all have higher $\mathrm{He} / \mathrm{H}$ than the universal value of 0.1 . The $\mathrm{N}$ and 0 abundance anomalies individually cannot be easily discerned from this

TABLE 2. Abundances of Ring Nebulae

\begin{tabular}{lllcccc}
\hline Object & Type & $\mathrm{He} / \mathrm{H}$ & $12+\log (\mathrm{O} / \mathrm{H})$ & $12+\log (\mathrm{N} / \mathrm{H})$ & $\log (\mathrm{N} / \mathrm{O})$ & $\mathrm{Ref}$. \\
\hline & & & & & & \\
NGC 6164-5 & Of & 0.117 & 8.25 & 8.13 & -0.12 & 1 \\
$\eta$ Car & LBV & 0.17 & $\leq 7.8$ & -9.0 & 1.2 & 2 \\
AG Car & LBV & $?$ & 7.2 & 7.5 & 0.3 & 3 \\
RCW 78 & WN & 0.11 & 8.92 & 8.07 & -0.85 & 4 \\
NGC 2359 & WN & 0.10 & 8.25 & 7.27 & -0.98 & 4 \\
NGC 3199 & WN & 0.095 & 8.51 & 7.55 & -0.96 & 5 \\
MR 100 & WN & 0.115 & 8.52 & 7.64 & -0.88 & 5 \\
MR 26 & WC & 0.09 & 8.55 & 7.55 & -1 & 5 \\
S308 & WN & 0.123 & 8.2 & 8.0 & -0.2 & 5 \\
RCW104 & WN & 0.15 & 8.54 & 7.85 & -0.69 & 5 \\
RCW58 & WN & 0.23 & 8.72 & 8.42 & -0.3 & 5 \\
NGC 6888 & WN & 0.188 & 8.15 & 8.45 & 0.3 & 6 \\
M1-67 & WN & 0.22 & 8.10 & 8.70 & 0.60 & 7 \\
& & & & & & \\
Sun & & 0.107 & 8.87 & 7.96 & -0.91 & 3 \\
MWG HII & & 0.1 & 8.7 & 7.57 & -1.13 & 3 \\
Type I PNe & & 0.135 & 8.71 & 8.88 & 0.17 & 3
\end{tabular}

Ref: 1. Dufour, Parker, and Henize 1988; 2. Davidson et al. 1986; 3. Mitra and Dufour 1990; 4. Esteban et al. 1990a; 5. Rosa and Mathis 1990; 6. Esteban and Vílchez 1990; 7. Esteban et al. 1990b. 
table, since the local interstellar abundances, subject to the galactic abundance gradient, are not listed. The N/O ratio, on the other hand, is normally quite constant with $\log (\mathrm{N} / \mathrm{O}) \sim-1$; any anomaly in $\mathrm{N} / 0$ ratio can be easily spotted. The nebulae that show enhanced $\mathrm{He} / \mathrm{H}$ all have $\mathrm{N} / \mathrm{O}$ ratio greater than the normal value.

A closer examination of the nebulae individually shows that the N/O increase is due to both an enhancement in $\mathrm{N}$ abundance and a depletion in 0 abundance. Dufour (1989b) has noticed that the sum of $\mathrm{N}$ and $\mathrm{O}$ is nearly constant in ring nebulae and compared them with the Type I planetary nebulae. For the Type I planetary nebulae, Feibelman et al. (1985) suggests that the reaction $0^{16}+2 \mathrm{p} \rightarrow \mathrm{N}^{14}+\alpha$ is responsible for converting $O$ into $N$. How about the massive stars? Nucleosynthesis models in massive stars need to explain these abundance anomalies.

The most severe depletion of 0 occurs in the nebulae around LBV's. In the $\eta$ Car ejecta, both $C$ and $O$ are depleted, while $N$ is enhanced; the sum of $C, N$, and $O$ seems to be conserved in $\eta$ Car ejecta (Dufour 1989a)! Unfortunately, most of the ring nebulae are not detectable in the UV carbon lines, and it is not possible to determine whether $(\mathrm{N}+0)$ or $(\mathrm{C}+\mathrm{N}+\mathrm{O})$ is constant. In either case, the nebular abundances place constraints on the stellar structure and nucleosynthesis models.

\subsection{Nebular Spectrophotometry and Stellar Effective Temperature ( $T_{\text {eff }}$ )}

The far UV energy distribution of a star cannot be observed directly; nevertheless, this information is carried in the surrounding nebula. By modelling the photoionization structure of a nebula to produce the observed nebular line fluxes and ratios, one can derive information on

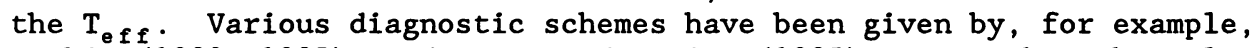
Mathis $(1982,1985)$, and Evans and Dopita (1985). Note that the value of $T_{\text {eff }}$ itself may have large uncertainties, since it depends on the stellar atmospheric models; nevertheless, the ranking of stellar $\mathrm{T}_{\text {eff }}$ within the same diagnostic scheme should be quite accurate.

Smith and Clegg (1990) find a temperature of 55,000-80,000 $\mathrm{K}$ for $\mathrm{HD}$ 50896 in S308. Esteban et a1. (1990a) find a temperature of $50,000 \mathrm{~K}$ for HD 56925 in NGC 2359 and $34,000 \mathrm{~K}$ for HD 117688 in RCW78. Rosa and Mathis (1990) use the abundance ratios of $\mathrm{S}^{+} / \mathrm{S}^{+2}$ and $0^{+} / 0$ as a diagnostic for $T_{\text {eff }}$ (Mathis 1982, 1985); they find S308, NGC 3199, NGC 6888 , and NGC 2359 are ionized by hot stars with $\mathrm{T}_{\text {eff }}$ between 60,000 and $90,000 \mathrm{~K}$, but RCW58, RCW104, MR26, and MR100 have such low excitation that their $\mathrm{T}_{\text {eff }}$ cannot be reliably determined. In general, the early WN's are hotter than the late WN's, and the WC6 star MR26 seems to be similar to or cooler than the late WN's. This result is quite consistent with the intuitive interpretation of the stellar spectra.

Emission lines from doubly ionized He, often seen in planetary nebulae with high excitation, are not expected in HII regions. However, at least 5 HII regions in the Local Group are known, before this symposium, to emit nebular HeII4686 line: G2.4+1.4 in the Galaxy, N44C and $\mathrm{N} 159 \mathrm{~F}$ in the LMC, N76 in the SMC, and IC1613\#3. G2.4+1.4 and IC1613\#3 are each ionized by a WO star, and the extended HeII emission is centered on the WO star (Dopita et al. 1990; Davidson and Kinman 1982). The positive association of nebular HeII emission with the wO 
stars implies that the effective temperature of wo stars can reach as high as 70,000-80,000 $\mathrm{K}$ ! This high temperature perhaps could have been expected, since WO stars emit strong OVI lines, and the hottest known planetary nebula nuclei also emit OVI lines. However, not every wo star is hot enough to produce doubly ionized He in the surrounding nebula, as Pakul1's (1990, private communication) search for HeII emission around other known wo stars (Barlow and Hummer 1982) yields no positive detection. $\mathrm{N} 159 \mathrm{~F}$ is ionized by the $\mathrm{X}$-ray binary LMC X-1 (Pakull and Angebault 1986). N44C is apparently centered on an 04-06 main sequence star (Stasinska, Testor, and Heydari-Malayeri 1986; Pakull and Motch 1989) and N76 centered on a WN3+O binary (Moffat 1988). A high stellar temperature for an 0 or WN star is quite surprising, and it has been suggested that $X$-rays and shock excitation may be responsible for the HeII emission (Pakull and Motch 1989; Chu and Mac Low 1990).

In this symposium, three new HeII4686 emitting nebulae are reported around WR stars: $\mathrm{Br} 2$ (WN1?) and Br40a (WN3+06) in the LMC, and AB-5 (WN3+WN6+0) in the SMC (Pakull 1990; Niemela 1990). Perhaps some early type WR stars are indeed hot!

\subsection{Ring Nebulae and Bubble Dynamics}

The W-type ring nebulae around WR stars (Chu 1981) were thought to be good wind-blown bubbles that can test theoretical models. When the momentum and energy conversion factors were found to deviate from those expected from Weaver et al.'s (1977) bubble models (Treffers and Chu 1982a; Chu 1982a), improved calculations including interstellar cloudlets have been carried out (McKee, Van Buren, and Lazareff 1984) and neutral material in the bubble shells has been proposed to explain the discrepancies (Van Buren 1986).

Now we know that some $W$-type nebulae actually contain shells of stellar ejecta. Their momentum and energy conversion factors would be quite meaningless because the shell kinetic energy is determined by stellar ejecta and has little to do with the total stellar wind energy output during the evolution of the shell. The discrepancy between the observed and bubble model predicted X-ray luminosities of NGC 6888 (Kähler, Ule, and Wendker 1987; Bochkarev 1988) can be well explained by the shell dynamics being dominated by the stellar ejecta. Even the rings without confirmed stellar ejecta may be contaminated by an unknown amount of stellar ejecta. Stellar winds interacting with the expanding circumstellar material lost by the progenitor cannot be described by the commonly-used model of a bubble in a stationary medium (Dyson and Smith 1985). The significance of these conversion factors may have been overrated; these factors have to be used with great caution.

\section{FUTURE WORK}

There are various scenarios regarding the evolution for massive stars. Since the abundances and kinematics of a ring nebula reflect the history of the stellar nucleosynthesis and mass loss, detailed studies of nebulae around different central stars may exclude or confirm some 
evolutionary scenarios. For example, if LBV's evolve into WN's, we must find some nebulae around LBVs that could be evolve into some rings around WN stars. Can a nebula like AG Car evolve into something like RCW58? Do their abundances agree? Are the nebular structures consistent with the evolution of stellar ejecta interacting with stellar winds? The answers to these questions rely on the accurate abundance determination and theoretical calculations of the nebular evolution in the future.

In order to make the aforementioned test, we need a large sample of ring nebulae around different types of massive stars. However, only one of star is known to have stellar ejecta, a couple of stars have filamentary bubbles, and 2-3 LBV's have resolved nebulae. Is this small number due to a lack of surveys and observations? Are of stars less efficient than WR stars in blowing bubbles and ejecting envelopes?

In the past, only cataloged WR stars or of stars are used to search for ring nebulae. The selection effect may not be as bad, if the ring nebulae in a galaxy is surveyed first, then the central stars are identified and classified. Our Galaxy is hopelessly opaque along the galactic plane, and distant galaxies would have resolution problem. The Magellanic Clouds are the most appropriate for a systematic survey of ring nebulae. A ring with 5 pc diameter will subtend nearly 20 " in the LMC, which can be easily resolved from the ground based telescopes. The central stars of ring nebulae can also be identified and classified. An extended survey for ring nebulae and central stars in the Magellanic Clouds is badly needed. Correlations between the nebular properties and the stellar types would be extremely interesting.

Abundance analysis of a ring nebula should try to differentiate the different components from the background. For example, both NGC 6888 and RCW58 have a shell of ejecta inside a bubble, and both nebulae have a stationary interstellar component beside the shell components. Does this stationary component have similar abundances as those in the shell components? Do the bubbles in NGC 6888 and RCW58 have abundances similar to those of the ejecta or the ambient medium?

The IRAS data can be used to search for ring features in the IR, but the interpretation may be tricky. A wind blown bubble is expected to be a soft X-ray source. ROSAT observations of ring nebulae should be made.

The author acknoledges the support of an International Travel Grant from the American Astronomical Society. This work is supported by NSF grant AST-8818192.

\section{REFERENCES}

Baars, J. W. M. and Wendker, H. J. 1987, Astr. Ap., 181, 210.

Barlow, M. J. and Hummer. D. G. 1982, in IAU Symposium No. 99, WolfRayet Stars: Observations, Physics, Evolution, eds. C. W. H. de Loore and A. J. Willis (Dordrecht: Reidel), p. 387.

Bochkarev, N. G. 1988, Nature, 332, 518.

Cappa de Nicolau, C. E., and Niemela, V. S. 1984, A. J., 89, 1398. 
Cappa de Nicolau, C. E., Niemela, V. S., and Arnal, E. M., 1986, A. J., 92, 1414 .

Cappa de Nicolau, C. E., Niemela, V. S., Dubner, G. M., and Arnal, E. M., 1988 , A. J., 96,1671 .

Chu, Y.-H. 1981, Ap. J., 249, 195. (Paper I)

Chu, Y.-H. 1982a, Ap. J., 254, 578. (Paper VI)

Chu, Y.-H. 1982b, Ap. J., $\underline{255}, 79$. (LMC paper II)

Chu, Y.-H. 1983a, Ap. J., 269, 202. (LMC paper III)

Chu, Y.-H. 1983b, Pub. A. S. P., 95, 873.

Chu, Y.-H. 1988, Pub. A. S. P., 100, 986.

Chu, Y.-H., Gull, T. R., Treffers, R. R., Kwitter, K. B., and Troland, T. H. 1982, Ap. J., 254, 562. (Paper IV)

Chu, Y.-H. and Lasker, B. M. 1980, Pub. A. S. P., 92, 730. (LMC paper I)

Chu, Y.-H. and Mac Low, M.-M. 1990, submitted to Ap. J.

Chu, Y. -H. and Treffers, R. R. 1981a, Ap. J., 249, 586. (Paper II)

Chu, Y.-H. and Treffers, R. R. 1981b, Ap. J., 250, 615. (Paper III)

Chu, Y.-H., Treffers, R. R., and Kwitter, K. B. 1983, Ap. J. Suppl., 53, 937. (Paper VIII)

Crampton, D. 1971, M. N. R. A. S., 153, 303.

Davidson, K., Dufour, R. J., Walborn, N. R., and Gull, T. R. 1986, Ap. J., 305, 867 .

Davidson, K. and Kinman, T. D. 1982, Pub. A. S. P., 94, 634 .

Deharveng, L., Israel, F. P., and Maucherat, M. 1976, Astr. Ap., 48, 63.

Dopita, M. A., Lozinskaya, T. A., McGregor, P. J., and Rawlings, S. J. 1990 , Ap. J., 351, 563.

Drissen, L., Shara, M. M., and Moffat, A. F. J. 1990, in this symposium.

Dubner, G. M., Niemela, V. S., and Purton, C. R. 1990, A. J., 99, 857.

Dufour, R. J. 1989a, Rev. Mexicana Astron. Astrof., 18, 87.

Dufour, R. J. 1989b, in IAU Symposium No. 131, Planetary Nebulae, ed.

S. Torres-Peimbert (Dordrecht: Kluwer), p. 216.

Dufour, R. J., Parker, R.A.R., and Henize, K. G. 1988, Ap. J., 327, 859.

Dyson, J. E. 1977, Ap. and Space Sci., 51, 197.

Dyson, J. E. and Ghanbari, J. 1989, Astr. Ap., 226, 270.

Dyson, J. E. and Smith, L. J. 1985, in Cosmic Gas Dynamics, ed. F. D. Kahn, VNU Science Press, Utrecht, Holland, p. 173.

Esteban, C., Vílchez, J. M., Manchado, A., and Edmunds, M. G. 1990a, Astr. Ap., 227, 515.

Esteban, C., Vílchez, J. M., Smith, L. J., and Manchado, A. 1990b, in preparation.

Evans, I. N. and Dopita, M. A. 1985, Ap. J. Suppl., 58, 125.

Feibelman, W., Aller, L. H., Keyes, C. D., and Czyzak, S. J. 1985, Proc. Nat1. Acad. Sci. USA, 2, 2202.

Georgelin, Y. M., Georgelin, Y. P., Laval, A., Monnet, G., and Rosado, M. 1983, Astr. Ap. Suppl., 54, 459.

González, J. and Rosado, M. 1984, Astr. Ap. (Letters), 134, L21.

Goudis, C. D., Meaburn, J., and Whitehead, M. J. 1988, Astr. Ap., 191,341 .

Gull, T. R. and Sofia, S. 1979, Ap. J., 230, 782 .

Heckathorn, J. N., Bruhweiler, F. C., and Gull, T. R. 1982, Ap. J., $\underline{252}, 230$.

Herbig, G. H. 1960, Ap. J. Supp1., 4, 337. 
Heydari-Malayeri, M., Melnick, J., and Van Drom, E. 1990, Astr. Ap., in press.

Jernigan, T. E. 1988, Ph.D. thesis, Rice University.

Johnson, H. M. 1975, Ap. J., 198, 111.

Johnson, H. M. 1982, Ap. J., 256, 559 .

Johnson, H. M. and Hogg, D. E. 1965, Ap. J., 142, 1033.

Kähler, H., Ule, T., and Wendker, H. J. 1987, Ap. Space Sci., 135, 105.

Kwitter, K. B. 1981, Ap. J., 245, 154.

Kwitter, K. B. 1984, Ap. J., 287, 840.

Leitherer, C. and Chavarria-K., C. 1987, Astr. Ap., 175, 208.

Lortet, M. C., Niemela, V. S., and Tarsia, R. 1980, Astr. Ap., 90, 210.

Lozinskaya, T. A. 1982, Ap. and Space Sci., 87, 313.

Lozinskaya, T. A. 1983, Sov. Astron. Lett., 9 , 247.

Lozinskaya, T. A., Lar'kina, V. V., and Putilina, E. V. 1984, Sov. Astron. Lett., $\underline{9}, 344$.

Marston, A. P. and Meaburn, J. 1988, M. N. R. A. S., 235, 391.

Mathis, J. S. 1982, Ap. J., 261, 195.

Mathis, J. S. 1985, Ap. J., 291, 247.

McCray, R. 1983, Highlights Astr., 6, 565.

McKee, C. F., Van Buren, D., and Lazareff, B. 1984, Ap. J. (Letters), $\underline{278}, \mathrm{~L} 115$.

Miranda, A.I. and Rosado, M. 1987, Rev. Mexicana Astr. Astrof., 14, 479.

Mitra, P. M. 1990, Ph.D. thesis, Rice University.

Mitra, P. M. and Dufour, R. J. 1990, M. N. R. A. S., 242, 98.

Moffat, A. F. J. 1988, Ap. J., 330, 776.

Nichols-Bohlin, J. and Fesen, R. A. 1990, in preparation.

Niemela, V. S. 1990, in this volume.

Pakul1, M. W. 1990, in this volume.

Pakul1, M. W. and Angebault, L. P. 1986, Nature, 322, 511.

Pakul1, M. W. and Motch, C. 1989, Nature, 337, 337.

Parker, R. A. R. 1978, Ap. J., 224, 873.

Pismis, P. 1974, Rev. Mexicana Astr. Astrof., 1, 45.

Rosa, M. R. and Mathis, J. S. 1990, Properties of Hot Luminous Stars: Boulder-Munich Workshop, ed. C. D. Garmany, Pub. A. S. P. Conf. Ser., 7, 135 .

Rosado, M. 1986, Astr. Ap., 160, 211.

Smith, L. F. 1967, Ph.D. thesis, Australian National University.

Smith, L. J. 1990, in this volume.

Smith, L. J. and Clegg, R. E. S. 1990, Properties of Hot Luminous Stars: Boulder-Munich Workshop, ed. C. D. Garmany, Pub. A. S. P. Conf. Ser., Z, 132 .

Solf, J. and Carsenty, U. 1982, Astr. Ap., 116, 54.

Stah1, 0. 1987, Astr. Ap., 182, 229.

Stah1, 0. 1989, in Physics of Luminous Blue Variables, eds. K. Davidson, A. F. J. Moffat, and H.J.G.L.M. Lamers (Dordrecht: Kluwer), p. 149.

Stahl, 0., Wolf, B., Klare, G., Cassatella, A., Krautter, J., Persi., P., and Ferrari-Toniolo, M. 1983, Astr. Ap., 127, 49.

Stasinnska, G., Testor, G., and Heydari-Malayeri, M. 1986, Astr. Ap. , 170 , L4.

Swings, J. P. and Preston, G. W. 1978, Ap. J., 220, 883.

Vílchez, J. M. and Esteban, C. 1990, in this volume. 
Treffers, R. R. and Chu, Y.-H. 1982a, Ap. J., 254, 569. (Paper V)

Treffers, R. R. and Chu, Y.-H. 1982b, Ap. J., 254, 132. (Paper VII)

Van Buren, D. 1986, Ap. J., 306, 538 .

Van Buren, D. and McCray, R. 1988, Ap. J. (Letters), 329, L93.

van der Hucht, K. A., Conti, P. S., Lundstrom, I., and Stenholm, B. 1981, Space Sci. Rev., 28, 227.

Walborn, N. R. 1982, Ap. J, 256, 452.

Walborn, N. R. and Blanco, B. M. 1988, Pub. A. S. P., 100, 797.

Walborn, N. R., Blanco, B. M., and Thackeray, A. D. 1978, Ap. J., $\underline{219}, 498$.

Weaver, H. 1977, "The Large-Scale Characteristics of the Galaxy", IAU

Symp. No. 84, ed. W. B. Burton et al. (Dordrecht: Reidel), p. 295.

Weaver, R., McCray, R. A., Castor, J., Shapiro, P., and Moore, R. 1977, Ap. J . , 218, 377 .

Wisotzki, L. and Wendker, H. J. 1989, Astr. Ap., 221, 311.

\section{DISCUSSION}

Moffat: If the WR nebulae that are stellar ejecta show enhanced $N / O$, the ejecta must come from the WR star itself: (1) Why then are the ejecta clumped or filamentary and, (2) why are they moving so slowly? When the ejecta left the observable part of the WR wind they must have had outward velocities of $v_{\infty} \approx(1-3) 10^{3} \mathrm{~km} \cdot \mathrm{s}^{-1}$. (3) If shot off like bullets, what slows them down? (4) Also, can the clumps be related to the blobs we see being ejected in the outer winds of WR stars?

Chu: (1) Stellar ejecta are subject to Rayleigh-Taylor instability, which makes the observed clumpy morphology. The ejecta may or may not be clumping initially. The ejecta in RCW104, the high velocity knots seen in [NII], may be clumping at the ejection. (2) Stellar ejecta and stellar wind result from different physical mechanisms, consequently, the ejection velocity may differ. The ejection velocity of the ejecta is probably of similar order as the escape velocity at the place where ejection occurs. Therefore, the ejection velocity would depend on the radius and mass of the star. (3) If ejecta are shot off like bullets, they will not only slow down gradually by sweeping up the ambient stationary medium, but they can be accelerated by the stellar wind, too.

Dopita: A high $[O I I I] / H \beta$ ratio is not necessarily due to shocks. Radiative shocks saturate at a value of the ratio determined by the $\mathrm{O}$ abundance. High $[O I I I] / H \beta$ ratios can be got by four mechanisms: (1) partially radiative shocks $(T[O I I I] \approx 40000 \mathrm{~K})$; (2) fast particle heating $(T[O I I I]$ unknown); (3) photoionisation at high specific radiation intensity $(T[O I I I] \lesssim 20000 K) ;(4) \mathrm{X}$-ray ionization $(T[O I I I]$ unknown).

Chu: The $[O I I I] / H \beta$ ratio is explained as partially radiative shocks by Dufour, Jerrigan, and Mitra for NGC 2359 and NGC 6888. The X-ray ionisation is unlikely as the X-ray emission comes from the bubble cavity while the $[O I I I]$ enhancement is on the outer surface of the shell.

de Groot: On the subject of stellar ejecta, low shocks and proper motions - where you said that you would not know what to do with such information - let me offer a suggestion. An idea of the velocity of the ejecta, when combined with their radii, gives you a timescale since their formation. This can then be combined with the space velocity - proper motion and radial velocity - of the "central" object to find a confirmation for the assumed velocity of ejecta. We did this in the case of P Cyg (1983) where it confirms the ejection of the 
radio feature some 30,000 years ago, i.e. in harmony with ejection at the red supergiant stage. P Cyg also has a much smaller circumstellar shell. In that case the size of the shell fits exactly its ejection some 400 years ago, i.e. at P Cyg's outburst in AD 1600 (Leitherer et al., 1987).

Drissen: In our M33 survey fields, which included $50 \mathrm{WR}$ stars, we found 11 good WR ring nebulae. We have to explain why about $75 \%$ of WR stars do not blow bubbles.

Chu: The small ones are probably not resolved at the distance of M33, hence have been missed.

Walborn: Davidson et al. had two indirect arguments that the $C$ depletion in the $\eta$ Car knots is not entirely due to grain formation: (1) $O$ is also depleted, but normal $S i$ abundance is observed and (2) as best could be determined, the absolute $N$ abundance is consistent with the normal sum of $C N O$.

Smith, Linda J.: I would like to make a comment on the ejecta nature of NGC 6888 . With WR ring nebulae, it is important to consider the effect of the progenitor wind on the ambient interstellar medium. For NGC 6888 this is particulary important because the central star HD 192163 is a member of the Cyg OBl association. It is therefore likely that the collective winds and supernovae from the association members will have evacuated the interstellar medium surrounding HD 192163 before the onset of the WR wind. From this then, NGC 6888 has to be composed of almost pure stellar ejecta.

Montmerle: Frequently, I have seen in the literature people deriving ages, e.g., from the radii...

Chu: It should not be called age, it should be called dynamic scale, radius over velocity, it is a convenient scale, it should not really be called age.

Montmerle: I just wanted to add that since in many cases you see that it is more momentum conserving, then energy conserving, then the actual age that you would deduce critically depends on the mechanism that you are assuming. People should watch out for these kinds of things, and even the dynamical age is in most of the cases useless.

Chu: It is a convenience scale, it has not much physical meaning.

Maeder: You say that the ejeca have higher N/O ratio, which is quite consistent with material processed by the CNO cycle and ejected before the WR phase or during the WN phase. But, what about material ejected during the WC phase, because then we would have the supposed strong oxygen and C-enrichment and do you have a signature of that effect?

Chu: No, we do not have an ejecta around WC stars. The ejecta is kind of tricky. Once it is ejected, it breaks up into small chunks. These small chunks will expand, the density goes down very quickly. The density goes down in some fashion, but the emission measure goes down the square of that, so the emission measure goes down quicker than the expansion and very quickly it disappears. It is a detection limit problem!

Smith, Lindsey: Following on the remark of Linda Smith, NGC 3199 and NGC 2359 are much more massive than NGC 6888 and RCW 58. The mechanisms involved are the same; only the density of the surround:ng ISM is higher, so the abundance anomalies of the WR wind are diluted by normal abundance of the swept up gas. 


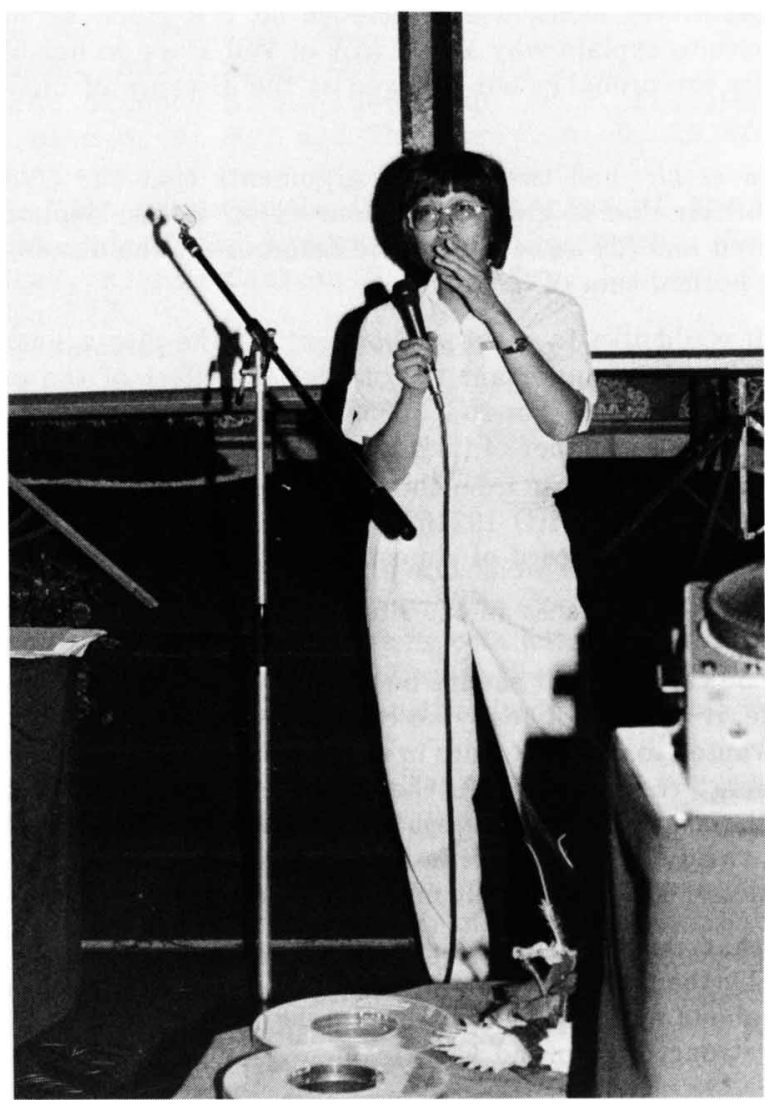

You-Hua Chu 